\section{Confirmation of risk of cancer in blepharocheilodontic syndrome}

Pathogenic variants in $\mathrm{CDH} 1$ were initially identified to be responsible for early-onset, multigenerational hereditary diffuse gastric cancer and lobular breast cancer (HDGC; OMIM 137215). In a small number of families affected with HDGC, cleft lip with or without cleft palate (CLP) has been found to segregate with the $C D H 1$ variant. ${ }^{1}$ Then, $C D H 1$ variants were identified in patients with nonsyndromic CLP (NSCLP) without history of diffuse gastric cancer (DGC) or lobular breast cancer (LBC). ${ }^{2}$ In HDGC, $C D H 1$ variant carriers have a lifetime high risk of DGC and LBC and the identification of a $C D H 1$ variant in a proband and his relatives leads to risk-reducing measures including prophylactic gastrectomy and annual breast magnetic resonance image (MRI). ${ }^{3}$

In 2017, we published the article entitled "Blepharocheilodontic syndrome is a $C D H 1$ pathway-related disorder due to variants in $C D H 1$ and CTNND1." ${ }^{\text {O }}$ Our team identified $\mathrm{CDH} 1$ heterozygous variants in seven patients from five families affected with blepharocheilodontic syndrome (BCDS1; MIM 119580) indicating that, beyond NSCLP, $\mathrm{CDH} 1$ variants could lead to a broader spectrum of developmental anomalies. ${ }^{4}$ A few months later, Kievit et al. confirmed these findings. ${ }^{5}$ In the light of both studies and the description of 26 patients from 18 families, variants causing BCDS1 were likely to localize in mutational hotspots, corresponding to E-cadherin calcium-binding sites in the EC1-EC2 linker domain and to exon 9-intron 9 junction, resulting in exon 9 skipping. They partially overlap with variants identified in NSCLP patients, such as p.(Asp254Asn) substitution. To date, this variant has never been associated with cancer, while it was identified in up to three BCDS1 and in two NSCPL large multigenerational families. ${ }^{2,5}$

Before the identification of $\mathrm{CDH} 1$ as the BCSD1-causing gene, it was not known that BCDS patients were at risk of cancer.

Recently, the first cases of DGC and LBC were reported in one BCDS1 family carrying variant c.768T $>A$ p.(Asn256Lys). ${ }^{6}$ This family showed variability in developmental anomalies ranging from NSCLP to classical BCDS1 facial features. ${ }^{6}$ In our previous work, we reported a patient and her daughter, both showing typical BCDS1 features and carrying the c.1320+1G>C p.(Tyr380_Ly440del) variation. ${ }^{4}$ This splice variant leads to exon 9 skipping and deletion of residues Tyr380 to Lys440. It was identified in nine patients from seven families, p.(Tyr380_Ly440del) representing therefore the most recurrent BCDS1-associated variant. ${ }^{4,5}$ The variant occurred de novo in all reported cases, except in our family. The segregation could not be performed in our proband's parents. While her mother did not harbor facial features of BCDS1, she died from fulminant gastric cancer at the age of 35 years. We were not able to retrieve the precise tumor type and genetic testing could not be carried out to confirm the hypothesis of a CDH1-associated DGC. The CDH1 variant was identified in the index BCDS1 patient when she was 40 years old. ${ }^{4}$ Because of this finding, together with the familial history of gastric cancer, preventive gastrostomy was discussed, but the patient preferred undergoing repetitive endoscopies with biopsies. When she was 43 years old, endoscopy showed a large ulcerated lesion at the greater curvature of the stomach. Pathology confirmed the diagnosis of signet ring cell carcinoma of the stomach. She underwent chemotherapy and gastrectomy. This clinical update confirms the risk of cancer in blepharocheilodontic syndrome.

In the invited commentary following our study, P.R. Benusiglio indicated that the involvement of $\mathrm{CDH} 1$ meant that BCDS1 families were likely at high risk of both DGC and $\mathrm{LBC}$, and that risk-reducing measures, however radical they might be, had to be discussed. ${ }^{7}$ In the case of both families showing BCDS1 and CDH1-related cancers, it is therefore evident that all $C D H 1$ variant carriers must benefit from preventive measures to reduce their risk of cancer, including prophylactic gastrectomy. But what can we advise to the other BCDS1 or NSCLP families? Identification of unexpected $\mathrm{CDH} 1$ variations through multigene panel testing results in a comparable dilemma: to recommend an invasive procedure with high morbidity and mortality to a family with little history of the disease or to leave untreated an invasive disease with high mortality. The authors ask for a better assessment of $C D H 1$ variant penetrance to better advise asymptomatic families regarding a preventive measure. ${ }^{8}$ Along the same line, further studies are needed to precisely determine the accurate penetrance of cancer in BCDS1. We now should address whether CDH1-related cancers cluster in some BCDS1 families, due to other familial genetic and environmental determinants, or whether the risk of cancer is instead linked to specific $\mathrm{CDH} 1$ variants.

\section{DISCLOSURE}

The authors declare no conflicts of interest.

Publisher's note Springer Nature remains neutral with regard to jurisdictional claims in published maps and institutional affiliations. 
Jamal Ghoumid, $M D, P h D$ (D) $^{1,2}$, Sophie Lejeune, $M D^{2}$, Florence Renaud, $M D, P h D^{3}$, Morgane Stichelbout, $M D^{1,3}$, Florence Petit, $\mathrm{MD}, \mathrm{PhD}^{1,2}$ and Sylvie Manouvrier-Hanu, $M D, P h D^{1,2}$

${ }^{1}$ Université de Lille, EA7364 RADEME, Lille, France; ${ }^{2} \mathrm{CHU}$ Lille, Clinique de Génétique "Guy Fontaine », Lille, France; ${ }^{3} \mathrm{CHU}$ Lille, Institut de pathologie, Lille, France. Correspondence: Jamal Ghoumid (jamal.ghoumid@chru-lille.fr)

\section{REFERENCES}

1. Frebourg $T$, Oliveira $C$, Hochain $P$, et al. Cleft lip/palate and $C D H 1 / E-$ cadherin mutations in families with hereditary diffuse gastric cancer. J Med Genet. 2006;43:138-142.

2. Brito LA, Yamamoto GL, Melo S, et al. Rare variants in the epithelial cadherin gene underlying the genetic etiology of nonsyndromic cleft lip with or without cleft palate. Hum Mutat. 2015:36:1029-1033.

3. van der Post RS, Vogelaar IP, Carneiro F, et al. Hereditary diffuse gastric cancer: updated clinical guidelines with an emphasis on germline $\mathrm{CDH} 1$ mutation carriers. J Med Genet. 2015;52:361-374.
4. Ghoumid J, Stichelbout M, Jourdain AS. et al. Blepharocheilodontic syndrome is a $\mathrm{CDH} 1$ pathway-related disorder due to mutations in $\mathrm{CDH} 1$ and CTNND1. Genet Med. 2017:19:1013-1021.

5. Kievit A, Tessadori F, Douben $\mathrm{H}$, et al. Variants in members of the cadherincatenin complex, $\mathrm{CDH1}$ and CTNND1, cause blepharocheilodontic syndrome. Eur J Hum Genet. 2018;26:210-219.

6. LeBlanc S, Naveen D, Haan E, et al. CDH1-related blepharocheilodontic syndrome is associated with diffuse gastric cancer risk. Am J Med Genet A. 2020 Apr 17; https://doi.org/10.1002/ajmg.a.61601 [Epub ahead of print].

7. Benusiglio PR. $C D H 1$ germline mutations: different syndromes, same management? Genet Med. 2017:19:965-966.

8. Huynh JM, Laukaitis CM. Panel testing reveals nonsense and missense $\mathrm{CDH} 1$ mutations in families without hereditary diffuse gastric cancer. Mol Genet Genomic Med. 2016;4:232-236.

Advance online publication 2 June 2020. doi:10.1038/s41436-020-0849-7 